\title{
Local Decay of Scattering Solutions to Schrödinger's Equation
}

\author{
Jeffrey Rauch`
}

Institute for Advanced Study, Princeton, New Jersey 08540, USA

\begin{abstract}
The main theorem asserts that if $H=\Delta+g V$ is a Schrödinger Hamiltonian with short range $V, \phi \in L_{\text {compact }}^{2}\left(\mathbb{R}^{3}\right)$, and $R>0$, then $\left\|\exp (i H t) \Pi_{S} \phi\right\|_{L^{2}(|x|<R)}=O\left(t^{-1 / 2}\right)$ as $t \rightarrow \infty$ where $\Pi_{S}$ is projection onto the orthogonal complement of the real eigenvectors of $H$. For all but a discrete set of $g, O\left(t^{-1 / 2}\right)$ may be replaced by $O\left(t^{-3 / 2}\right)$.
\end{abstract}

\section{§1. Introduction}

A basic dynamical equation in nonrelativistic quantum mechanics is Schrödinger's equations for $u(t, x)$ :

$$
\frac{1}{i} \frac{\partial u}{\partial t}=\sum_{i=1}^{3} \frac{\partial^{2} u}{\partial x_{i}^{2}}+V(x) u
$$

where $t \in \mathbb{R}$ and $x \in \mathbb{R}^{3}$. Let $H_{0} \equiv \Delta \equiv \sum \frac{\partial^{2}}{\partial x_{i}^{2}}$ and $H \equiv \Delta+V$. The formal solution of (1.1) is $u(t)=e^{i H t} u(0)$. If $V$ is real valued and not too singular too example if $\left.V \in L_{2}\left(\mathbb{R}^{3}\right)\right)$, then $H$ with domain the Sobolev space $W_{2}\left(\mathbb{R}^{3}\right)$ is selfadjoint on $L_{2}\left(\mathbb{R}^{3}\right)$ so $e^{i H t}$ defines a one parameter group of unitary maps. Two types of solution are easily visualized: bound states, $e^{i \omega t} \phi(x)$, for which $|u(t, x)|$ is independent of time, and, scattering solutions $u(t, x)$ with the property that for any ball $\mathscr{B}$ in $\mathbb{R}^{3}$

$$
\int_{\mathscr{B}}|u(t, x)|^{2} d x \rightarrow 0 \quad \text { as } \quad t \rightarrow \infty .
$$

If $V(x) \rightarrow 0$ as $|x| \rightarrow \infty$, it is natural to expect that for solutions in the latter class there is a $u_{+}(t)=e^{i t H_{0}} u_{+}(0)$ with $\left\|u(t)-u_{+}(t)\right\|_{L^{2}\left(\mathbb{R}^{3}\right)} \rightarrow 0$ as $t \rightarrow \infty$. There is a large and rich literature devoted to showing that these two types of solution form an exhaustive list, that is, $L_{2}\left(\mathbb{R}^{3}\right)$ can be written as an orthogonal direct sum $\mathscr{H}_{B} \oplus \mathscr{H}_{S}$ such that $e^{i t H}$ maps both $\mathscr{H}_{B}$ and $\mathscr{H}_{S}$ into themselves, $\mathscr{H}_{B}$ is spanned by eigenvectors of $H$, and $\mathscr{H}_{S}$ consists of solutions which decay locally in the sense of (1.2). For details see $([1,5,6,13])$.

* Research supported by the National Science Foundation under grants NSF GP 34260 and MCS 72-05055 A04 
This paper is devoted to obtaining more detailed information about the scattering solutions $e^{i t H} \uparrow \mathscr{H}_{\mathrm{S}}$. If one observes from a fixed position in space, then the solutions decay to zero. The question we ask is at what rate does the solution decay. To motivate the result, consider the free motion $u_{\mathrm{free}}=e^{i t H_{0}} \psi$. The solution is given by the integral

$$
\frac{c}{t^{3 / 2}} \int e^{i|x-y|^{2} / 4 t} \psi(y) d y
$$

where $c=(4 \pi i)^{-3 / 2}$. It follows that for $\psi \in L_{1}\left(\mathbb{R}^{3}\right)$

$$
\left|u_{\text {free }}(t, x)\right| \leqq|c|\|\psi\|_{L_{1}} t^{-3 / 2}
$$

decays like $t^{-3 / 2}$. For $\psi \in L_{2}\left(\mathbb{R}^{3}\right)$ the solution is not continuous and pointwise estimates are neither natural nor true. However, one might expect that $\left\|u_{\text {free }}(t)\right\|_{L_{2}(\mathscr{B})}=O\left(t^{-3 / 2}\right)$ for any bounded set $\mathscr{B}$. This also is false. In fact, there is no positive function $f(t)$ with $f(t) \rightarrow 0$ as $t \rightarrow \infty$ such that $\left\|u_{\text {free }}(t)\right\|_{L_{2}(\mathscr{B})}=O(f(t))$ as $t \rightarrow \infty$ for all $\psi \in L_{2}\left(\mathbb{R}^{n}\right)$. To see this, consider the map $A(t): \psi \mapsto\left(f(t)^{-1} e^{i t H_{0}} \psi\right) \uparrow \mathscr{B}$. If the asserted local decay were true then for any $\psi \in L_{2}$, the set $\{A(t) \psi: t \geqq 0\}$ would be bounded in $L_{2}(\mathscr{B})$ and the uniform boundedness principle would imply that $\{A(t): t \geqq 0\}$ is bounded in $\operatorname{Hom}\left(L_{2}\left(\mathbb{R}^{3}\right), L_{2}(\mathscr{B})\right)$ so there would be a constant $c>0$ such that for all $t \geqq 0$

$$
\left\|u_{\text {f ree }}(t)\right\|_{L_{2}(\mathscr{B})} \leqq c f(t)\|\psi\|_{L_{2}\left(\mathbb{R}^{n}\right)}
$$

Choose $T$ so large that $c f(T)<1$. For $\varphi \in C_{0}^{\infty}(\mathscr{B})$, let $\psi=e^{-i T H_{0}} \varphi$. Then $\left\|u_{\text {free }}(T)\right\|_{L_{2}(\mathscr{B})}=\|\varphi\|_{L_{2}\left(\mathbb{R}^{3}\right)}$ violating (1.3). Physically, this corresponds to preparing a solution that starts near infinity and arrives at $\mathscr{B}$ only after a long interval of time has elapsed. To get a quantitative form of (1.2), one must restrict to initial states which are small at infinity. For example, for any $\mathscr{B}$ we have

$$
\left\|u_{\text {free }}(t)\right\|_{L_{2}(\mathscr{B})} \leqq c|t|^{-3 / 2}\left\|u_{\text {free }}(0)\right\|_{L^{2}\left(\mathbb{R}^{n}\right)}
$$

for all $u_{\text {free }}(0)$ supported in a compact set $K$. The constant $c$ depends on $K$ and $\mathscr{B}$ but not on $u_{\text {free }}(0)$.

A stronger inequality than (1.4) is derived as follows. Choose a function $\varrho$ such that

$$
\varrho \in C^{\infty}\left(\mathbb{R}^{3}\right) \text { and } \varrho(x)=|x| \text { for }|x| \text { large. }
$$

For $\varepsilon>0$, let $E_{\varepsilon}$ be the multiplication operator

$$
E_{\varepsilon} \varphi=e^{-\varepsilon \varrho} \varphi \text {. }
$$

The operator $t^{3 / 2} E_{\varepsilon} e^{i t H_{0}} E_{\varepsilon}$ has kernel

$$
c e^{-\varepsilon \varrho(y)} e^{i|x-y|^{2} / 4 t} e^{-\varepsilon \varrho(x)}
$$

which is bounded in $L_{2}\left(\mathbb{R}^{3} \times \mathbb{R}^{3}\right)$ independent of $t>0$. Thus

$$
\left\|E_{\varepsilon} e^{i t H_{0}} E_{\varepsilon}\right\|_{\operatorname{Hom}\left(L_{2}\left(\mathbb{R}^{3}\right)\right)} \leqq c_{\varepsilon} t^{-3 / 2} .
$$

In terms of the solutions $u$ this asserts that

$$
\left\|e^{-\varepsilon \varrho} u_{\text {free }}(t)\right\|_{L_{2}} \leqq c_{\varepsilon}|t|^{-3 / 2}\left\|e^{\varepsilon \varrho} u_{\text {free }}(0)\right\|_{L_{2}}
$$


provided the right hand side is finite. Inequality (1.7) implies (1.4). Our main result generalizes (1.6) to the operators $e^{i t H}$.

By analogy with (1.7), it is natural to expect that scattering solutions of the perturbed equation decay like $t^{-3 / 2}$. The correct rate is $t^{-1 / 2}$. This is the content of Theorem 1 which holds even when $H$ is not selfadjoint. Recall that if $V \in L_{2}\left(\mathbb{R}^{3}\right)$, $\operatorname{Im} V \geqq 0$, and $H=\Delta+V$ with domain equal to the Sobolev space $W_{2}\left(\mathbb{R}^{3}\right)$, then $i H$ is a maximal dissipative operator on $L_{2}\left(\mathbb{R}^{3}\right)$ (see $\S 2$ for details) so $e^{i t H}$ is a contraction semigroup.

Theorem 1. Suppose that for some $\varepsilon>0$ and $p>2, e^{2 \varepsilon|x|} V \in L_{p}\left(\mathbb{R}^{3}\right)$ and $\operatorname{Im} V \geqq 0$. Let $\mathscr{H}_{B} \subset L_{2}\left(\mathbb{R}^{3}\right)$ be the (finite dimensional) set of eigenvectors of the operator $H=\Delta$ $+V$ with real eigenvalues $\mathscr{H}_{S} \equiv \mathscr{H}_{B}^{\perp}$ and $\Pi_{S}$ be orthogonal projection onto $\mathscr{H}_{S}$. Then $\mathscr{H}_{B}$ and $\mathscr{H}_{S}$ are invariant subspaces for $e^{i t H}$ and there is a constant $c$ such that

$$
\left\|E_{\varepsilon} e^{i t H} \Pi_{S} E_{\varepsilon}\right\|_{H o m\left(L_{2}\left(\mathbb{R}^{3}\right)\right)} \leqq c(1+t)^{-1 / 2}
$$

for all $t \geqq 0$, where $E_{\varepsilon}$ is the operator multiplication by $e^{-\varepsilon \varrho}$ and $\varrho$ is described in (1.5). In particular, if $u(t)=e^{i t H} u(0)$ and $e^{\varepsilon} u(0) \in L_{2}\left(\mathbb{R}^{3}\right)$, then

$$
\left\|e^{-\varepsilon \varrho} \Pi_{S} u(t)\right\|_{L_{2}\left(\mathbb{R}^{3}\right)} \leqq c(1+t)^{-1 / 2}\left\|e^{\varepsilon \varrho} u(0)\right\|_{L_{2}\left(\mathbb{R}^{3}\right)} .
$$

Examples. If $V(x)=|x|^{-\alpha} F(x) e^{-a|x|}$ with $a>0$ and $F \in L_{\infty}\left(\mathbb{R}^{3}\right)$, then $V$ satisfies the hypotheses of the theorem provided $\alpha<3 / 2$. If $\alpha=1$ then $V$ satisfies the hypotheses if $F \in L_{p}\left(\mathbb{R}^{3}\right)$ for some $p>6$. These potentials are Yukawian in form.

The exponential decay of $V$ is essential for our method. On the other hand, we have not been economical in our use of the decay. I expect that the same conclusions are true provided $e^{\varepsilon \varrho} V \in L_{p}$ for some $p>2$. No doubt, potentials with more severe local singularities could also be permitted.

On the other hand, the $t^{-1 / 2}$ rate is sharp. There is a $B \in \operatorname{Hom}\left(L_{2}\right)$ such that

$$
\left\|E_{\varepsilon} e^{i t H} \Pi_{S} E_{\varepsilon}-t^{-1 / 2} B\right\|_{\operatorname{Hom}\left(L_{2}\left(\mathbb{R}^{3}\right)\right)} \leqq c t^{-3 / 2}
$$

and for some potentials, $B$ is not zero. In fact, there is an asymptotic expansion for $E_{\varepsilon} e^{i t H} \Pi_{S} E_{\varepsilon}$ in decreasing powers of $t$ with $t^{-1 / 2} B$ as the leading term. Equation (1.10) shows that scattering solutions decay like $t^{-3 / 2}$ if and only if $B=0$. We will show that for most potentials this is the case. For example, if $H=\Delta+g V$ with $V$ as in Theorem 1 and $g \in \mathbb{R}$, then for all but a discrete set of values of $g$, the scattering solutions decay like $t^{-3 / 2}$. A related result is that if $V \leqq 0$ then $B=0$ so for nonpositive potentials the $t^{-3 / 2}$ rate always holds. On the other hand, $B \neq 0$ at those values of the coupling constant at which an eigenvalue emerges from the continuous spectrum. In particular, $B \neq 0$ if nullspace $(H) \neq\{0\}$. The results described in this paragraph are contained in $\S 4$.

Theorem 1 is proved by Laplace transform methods analogous to those which have recently been applied to study the asymptotic behavior of hyperbolic partial differential equations $([16,11])$. The basic idea is to obtain an analytic continuation of the resolvent of $H$ across the spectrum of $H$ and then to shift the contour in the inverse Laplace transform representation for $E_{\varepsilon} e^{i t H} E_{\varepsilon}$. A critical ingredient is an estimate for $E_{\varepsilon}\left(\lambda-H_{0}\right)^{-1} E_{\varepsilon}$ as $\operatorname{Re} \lambda \rightarrow-\infty$ (high energy estimates). These estimates are used to justify the Laplace inversion. Our derivation of these estimates is novel. It depends on Huyghens' principle for the wave equation 
$u_{t t}-H_{0} u=0$. Though there are certainly other ways to prove the inequalities, I think that this method is particularly interesting. Since the solutions of the wave equation have a great deal of structure (finite speed, Huyghens' principle, geometric optics, ... etc.), I feel that the solutions of the equations $u_{t t}-H_{0} u=0$ and $u_{t t}-H u=0$ should be rich sources of information about $H$ and $H_{0}$. The derivation of high energy resolvent estimates is one example. The resolvent inequalities are proved in $\S 2$ and are used to prove Theorem 1 in $\S 3$.

There is another not unrelated approach to the problem of local decay (see $[7,8]$ ) which begins with the observation that the function which vanishes for $t<0$ and is equal to $e^{-\varepsilon t} e^{i t H} \varphi$ for $t \geqq 0$, has Fourier transform $\lambda \mapsto(2 \pi)^{-1 / 2}(\lambda+i \varepsilon+H)^{-1} \varphi$. Thus Parseval's theorem implies that for $A \in \operatorname{Hom}\left(L_{2}\left(\mathbb{R}^{3}\right)\right)$

$$
\int_{0}^{\infty} e^{-2 \varepsilon t}\left\|A e^{i t H} \varphi\right\|^{2} d t=\frac{1}{2 \pi} \int_{-\infty}^{\infty}\left\|A(\lambda+i \varepsilon+H)^{-1} \varphi\right\|^{2} d \lambda .
$$

For suitably "smooth" or "gentle" operators $A$ and initial states $\varphi$, one shows that the integrand on the right hand side has a limit as $\varepsilon \rightarrow 0$ and that

$$
\int_{0}^{\infty}\left\|A e^{i t H} \varphi\right\|^{2} d t=\frac{1}{2 \pi} \int_{-\infty}^{\infty}\left\|A(\lambda+i 0+H)^{-1} \varphi\right\|^{2} d \lambda .
$$

In this way one can show that $A e^{i t H} \varphi$ is square integrable in time which is a decay theorem. Lavine [8] has given sharp results of this sort. In a sense, our method corresponds to going beyond the limit $\varepsilon=0$ to $\varepsilon<0$ in the above argument.

In the final section of the paper we discuss the behavior of scattering solutions to Schrödinger's equation for "intermediate" size times. Here terms of the form $e^{\tau_{j} t} \varphi(x)$ with $\operatorname{Re} \tau_{j}<0$ play an important role. The numbers $-i \tau_{j}$ are poles of the analytic continuation of the resolvent of $H$ across the spectrum of $H$. If $\operatorname{Re} \tau_{j}$ is small, these solutions are long lived. In the physical literature (see [3, Chapter 8]), this behavior is called a resonance. We give a rigorous justification of the occurrence of such terms in the local behavior of solutions to Schrödinger's equation and of their connection with singularities of the analytically continued resolvent.

Acknowledgement. It is a pleasure to thank G. Kaylor for her support and encouragement and I. Herbst, L. Gårding, and P. Deift for stimulating conversations about this research.

\section{§ 2. Resolvent Estimates}

The idea of the proof of Theorem 1 is to write

$$
E_{\varepsilon} e^{i t H} E_{\varepsilon}=\frac{1}{2 \pi i} \int_{d-i \infty}^{d+i \infty} e^{\tau t} E_{\varepsilon}(\tau-i H)^{-1} E_{\varepsilon} d t
$$

for $d>0$ and to shift the contour of integration into the halfplane $\operatorname{Re} \tau<0$. For this we must analytically continue the truncated resolvent across the spectrum of $i H$ and obtain estimates which justify the convergence of the integral in (2.1) and the deformation using Cauchy's theorem. We begin with a review of the basic properties of $H$. 
Let $H_{0}=\Delta=\sum_{i=1}^{3} \frac{\partial^{2}}{\partial x_{i}^{2}}$ be the operator on $L_{2}\left(\mathbb{R}^{3}\right)$ with domain, $\mathscr{D}\left(H_{0}\right)$, the Sobolev space $W_{2}\left(\mathbb{R}^{3}\right)=\left\{f \in L_{2}\left(\mathbb{R}^{3}\right): D_{x}^{\alpha} f \in L_{2}\left(\mathbb{R}^{3}\right)\right.$ for $\left.|\alpha| \leqq 2\right\}$. Then $H_{0}$ is selfadjoint and spectrum $\left(H_{0}\right)=(-\infty, 0]$. The reader is warned that this differs from the standard convention which has $H_{0}=-\Delta$ so that $H$ and $H_{0}$ are bounded below. The two conventions are mathematically equivalent one arising from the other by either reversing time or by replacing $i$ by $-i$ (Note that there is no canonical way of choosing a square root of -1.). Suppose that $V \in L_{2}\left(\mathbb{R}^{3}\right)$, then $V$ is a small perturbation of $H_{0}$ in the sense that $\mathscr{D}(V) \supset \mathscr{D}\left(H^{0}\right), V\left(\kappa-H_{0}\right)^{-1}$ is a compact operator on $L_{2}\left(\mathbb{R}^{3}\right)$ for $\kappa \notin \operatorname{spectrum}\left(H_{0}\right)$ and $\left\|V(\kappa-\Delta)^{-1}\right\| \rightarrow 0$ as distance $\left(\kappa, R_{-}\right) \rightarrow \infty($ see $[14$, p. 32]).

It follows that $H=H_{0}+V$ with $\mathscr{D}(H)=\mathscr{D}\left(H_{0}\right)$ is closed and if dist $\left(\kappa, \mathbb{R}_{-}\right)>c_{1}$, then $\kappa$ is in the resolvent set of $H$ and the following resolvent identities hold.

$$
\begin{aligned}
& (\varkappa-H)^{-1}=\left(x-H_{0}\right)^{-1}\left[I+V\left(x-H_{0}\right)^{-1}\right]^{-1} \\
& \left(\varkappa-H_{0}\right)^{-1}-(\varkappa-H)^{-1}=(x-H)^{-1} V\left(x-H_{0}\right)^{-1} .
\end{aligned}
$$

From (2.2) we see that if $\operatorname{dist}\left(\varkappa, \mathbb{R}_{-}\right) \geqq c_{2}$, then

$$
\left\|(x-H)^{-1}\right\| \leqq 2 \operatorname{dist}\left(x, \mathbb{R}_{-}\right)^{-1} \text {. }
$$

To make the next computations more transparent, we introduce a table of notations.

\begin{tabular}{ll}
\hline Symbol & Meaning \\
\hline$R(z), R^{0}(z)$ & $(z-H)^{-1},\left(z-H_{0}\right)^{-1}, z \notin$ spectrum \\
$E_{\varepsilon}$ & The operator multiplication by $e^{-\varepsilon \varrho}$ \\
$R_{\varepsilon}(z), R_{\varepsilon}^{0}(z)$ & $E_{\varepsilon} R(z) E_{\varepsilon}, E_{\varepsilon} R^{0}(z) E_{\varepsilon}$ \\
$L_{p}, W_{2}$ & $L_{p}\left(\mathbb{R}^{3}\right), W_{2}\left(\mathbb{R}^{3}\right)$ \\
\|\|$_{X, Y}$ & Norm in Hom $(X, Y)$ when $X$ and $Y$ are Banach spaces \\
Hom $(X)$ & Hom $(X, X)$ \\
$\operatorname{Kernel}_{\varepsilon}$ & The distribution kernel of an operator, never the nullspace \\
$F_{\varepsilon}^{0}(\zeta), F_{\varepsilon}(\zeta)$ & Analytic continuations of $R_{\varepsilon}^{0}\left(\zeta^{2}\right)$ and $R_{\varepsilon}\left(\zeta^{2}\right)$ \\
$G_{\varepsilon}(\tau)$ & Analytic continuation of $-i R_{\varepsilon}(-i \tau)$ (see end of $\left.\S 2\right)$ \\
\hline
\end{tabular}

The operator $R^{0}(z)$ is defined for $z$ in the slit plane $\mathbb{C} \backslash \mathbb{R}$. It will be easier to study $R^{0}\left(\zeta^{2}\right)$ which is defined for $\operatorname{Re} \zeta>0$ and has distribution kernel

$$
\frac{e^{-\zeta|y-x|}}{4 \pi|y-x|} \text {. }
$$

Lemma 1. For any $\varepsilon>0$, the function $\zeta \mapsto E_{\varepsilon} R^{0}\left(\zeta^{2}\right) E_{\varepsilon}$ extends to an analytic function on the set $\operatorname{Re} \zeta>-\varepsilon$ with values in the compact operators on $L_{2}$.

Proof. For $\operatorname{Re} \zeta>0$, the kernel of $R_{\varepsilon}^{0}\left(\zeta^{2}\right)$ is given by

$$
\frac{e^{-\varepsilon \varrho(y)} e^{-\zeta|y-x|} e^{-\varepsilon \varrho(x)}}{4 \pi|y-x|}
$$

which for $\operatorname{Re} \zeta>-\varepsilon$ is a holomorphic function of $\zeta$ with values in $L_{2}\left(\mathbb{R}^{3} \times \mathbb{R}^{3}\right)$. 
The next result, due to Dolph, McLeod, and Thor [2] shows that a similar assertion is true for $R_{\varepsilon}\left(\zeta^{2}\right)$.

Lemma 2. Suppose that $e^{\varepsilon \varrho} V \in L_{2}$ for some $\varepsilon>0$. Then, the function $\zeta \mapsto R_{\varepsilon}\left(\zeta^{2}\right)$ from $\operatorname{Re} \zeta>\sqrt{c}$ to $\operatorname{Hom}\left(L_{2}\right)$ extends to a meromorphic function of $\zeta$ on $\operatorname{Re} \zeta>-\varepsilon$ with values in the compact operators on $L_{2}$.

Proof. Multiply (2.3) on the left and the right by $E_{\varepsilon}$ to obtain for $\operatorname{Re} \zeta>\sqrt{c}$

$$
R_{\varepsilon}\left(\zeta^{2}\right)\left[I+\left(e^{\varepsilon \varrho} V\right) R^{0}\left(\zeta^{2}\right) E_{\varepsilon}\right]=R_{\varepsilon}^{0}\left(\zeta^{2}\right) .
$$

The operator in brackets is equal to $I+K(\zeta)$ where $K(\zeta)$ has the kernel

$$
K(\zeta, y, x)=\frac{e^{\varepsilon \varrho(y)} V(y) e^{-\zeta|y-x|} e^{-\varepsilon \varrho(x)}}{4 \pi|y-x|}
$$

which is holomorphic on $\operatorname{Re} \zeta>-\varepsilon$ with values in $L_{2}\left(\mathbb{R}^{3} \times \mathbb{R}^{3}\right)$. Thus $K(\zeta)$ is holomorphic on the same set with values in the compact operators on $L_{2}$. In addition, $\|K(\zeta)\|_{\text {Hilbert-Schmidt }} \rightarrow 0$ as $\operatorname{Re} \zeta \rightarrow+\infty$ so that $[I+K(\zeta)]^{-1}$ exists for $\operatorname{Re} \zeta$ large. Thus the analytic Fredholm theorem [12, Theorem VI.14] together with Lemma 1 implies that

$$
R_{\varepsilon}\left(\zeta^{2}\right)=R_{\varepsilon}^{0}\left(\zeta^{2}\right)[I+K(\zeta)]^{-1}
$$

has a meromorphic continuation to $\operatorname{Re} \zeta>-\varepsilon$ with values in the compact operators on $L_{2}$.

It is dangerous to continue to use the notation $R_{\varepsilon}^{0}\left(\zeta^{2}\right)$ for $\operatorname{Re} \zeta<0$ since there would be two possible meanings: one as the analytic continuation and another as $R_{\varepsilon}^{0}$ evaluated at the point $\zeta^{2} \notin \operatorname{spectrum}\left(H_{0}\right)$. The symbol $R_{\varepsilon}^{0}\left(\zeta^{2}\right)$ will be used only in the second sense. For the analytic continuation a different symbol is used.

Definition. Let $F_{\varepsilon}^{0}(\zeta)$ be the analytic function from $\operatorname{Re} \zeta>-\varepsilon$ to $\operatorname{Hom}\left(L_{2}\right)$ such that $F_{\varepsilon}^{0}(\zeta)=R_{\varepsilon}^{0}\left(\zeta^{2}\right)$ for $\operatorname{Re} \zeta>0$. Let $F_{\varepsilon}:\{\operatorname{Re} \zeta>-\varepsilon\} \rightarrow \operatorname{Hom}\left(L_{2}\right)$ be the meromorphic function with $F_{\varepsilon}(\zeta)=R_{\varepsilon}\left(\zeta^{2}\right)$ for $\operatorname{Re} \zeta$ large.

The next goal is to show that $F_{\varepsilon}(\zeta)$ is small when $\operatorname{Im} \zeta$ is large. The basis of the method is the representation

$$
F_{\varepsilon}(\zeta)=F_{\varepsilon}^{0}(\zeta)[I+K(\zeta)]^{-1}
$$

which is merely a restatement of (2.9). Observe that

$$
K(\zeta)=\left(e^{2 \varepsilon \varrho} V\right) F_{\varepsilon}^{0}(\zeta)
$$

so it is natural to expect that $F_{\varepsilon}(\zeta)$ is small wherever $F_{\varepsilon}^{0}(\zeta)$ is small. For $\operatorname{Im} \zeta$ very large, the kernel $(2.6)$ of $F_{\varepsilon}^{0}(\zeta)$ is highly oscillatory which forces the norm to be small.

Proposition 3. For any $a>0$ there is a constant $c=c(a, \varepsilon)$ such that

$$
\begin{aligned}
& \left\|F_{\varepsilon}^{0}(\zeta)\right\|_{L_{2}, L_{2}} \leqq \frac{c}{1+|\zeta|}, \\
& \left\|F_{\varepsilon}^{0}(\zeta)\right\|_{L_{2}, L_{\infty}} \leqq c
\end{aligned}
$$

for all $\zeta$ with $\operatorname{Re} \zeta>-\frac{\varepsilon}{2}+a$. 
For $\zeta \in i \mathbb{R}$ such estimates were proved by Herbst [4, Appendix]. The estimates (2.12) and (2.13) are sharp in the sense that the rates of decay as $|\operatorname{Im} \zeta| \rightarrow \infty$ are correct. In fact, $\left\|F_{\varepsilon}^{0}(i x)\right\|_{L_{2}, L_{2}} \geqq$ const $|x|^{-1}$ for $|\kappa|>1$, and $\left\|F_{\varepsilon}^{0}(\zeta)\right\|_{L_{2}, L_{\infty}}$ is independent of $\operatorname{Im} \zeta$. The second assertion is elementary and is proved below. A proof of the first assertion is contained in an appendix at the end of this section. On the other hand, the result is not sharp in the sense that estimate (2.14) should be valid in any region $\operatorname{Re}>-\varepsilon+a$. It is in the proof of (2.14) that the wave equation $u_{t t}-H_{0} u=0$ enters.

Proof of (2.13).Using the expression (2.8) for the kernel of $F_{\varepsilon}^{0}(\zeta)$, one finds

$$
\left\|F_{\varepsilon}^{0}(\zeta)\right\|_{L_{2}, L_{\infty}}^{2}=\sup _{x \in \mathbb{R}^{3}} \int \frac{e^{-2 \varepsilon \varrho(y)} e^{-2 \operatorname{Re} \zeta|y-x|} e^{-2 \varepsilon \varrho(x)}}{(4 \pi|y-x|)^{2}} d y
$$

which as asserted after the statement of Proposition 3 is independent of the imaginary part of $\zeta$. Choose $c_{1}$ (possibly negative) so that $\varrho(x) \geqq c_{1}+|x|$. Then

$$
\left\|F_{\varepsilon}^{0}(\zeta)\right\|_{L_{2}, L_{\infty}}^{2}<\sup _{x} e^{-4 c_{1}} \int \frac{e^{-2 \varepsilon|y|} e^{-2 \operatorname{Re} \zeta|y-x|} e^{-2 \varepsilon|x|}}{(4 \pi|y-x|)^{2}} d y .
$$

The supremum occurs when $x=0$ and the resulting expression implies (2.13) for $\operatorname{Re} \zeta>-\varepsilon+a$.

Proof of (2.13). Consider the Cauchy problem

$$
w_{t t}-\Delta w=0, \quad w(0)=f, \quad w_{t}(0)=0
$$

and let $P(t)$ be the solution operator, $P(t) f=w(t)$. Then $P(t)=\cos t \sqrt{-H_{0}}$ so $\|P(t)\|_{L_{2}, L_{2}}=1$. Since

$$
\int_{0}^{\infty} e^{-\zeta t} \cos t \lambda d t=\frac{\zeta}{\zeta^{2}+\lambda^{2}}, \quad \operatorname{Re} \zeta>0,
$$

it follows that

$$
\int_{0}^{\infty} e^{-\zeta t} P(t) d t=\zeta R^{0}\left(\zeta^{2}\right), \quad \operatorname{Re} \zeta>0
$$

This formula can also be derived by taking the Laplace transform of (2.14) directly. If $P(t, y, x)$ is the kernel of $P(t)$ then Huyghen's principle asserts that $\operatorname{supp} P(t, y, x) \subset\{(y, x)|| y-x \mid=t\}$. In particular, if $\operatorname{supp} f \subset\{|x| \leqq t / 2\}$ then $\operatorname{supp} P(t) f \subset\{|x| \geqq t / 2\}$. Formula (2.15) implies that for $\operatorname{Re} \zeta>0$

$$
F_{\varepsilon}^{0}(\zeta)=\frac{1}{\zeta} \int_{0}^{\infty} e^{-\zeta t} E_{\varepsilon} P(t) E_{\varepsilon} d t
$$

To prove the proposition, it suffices to show that for $t$ large,

$$
\left\|E_{\varepsilon} P(t) E_{\varepsilon}\right\|_{L_{2}, L_{2}} \leqq c\left\|E_{\varepsilon}\right\|_{L_{2}, L_{2}} e^{-\varepsilon t / 2}
$$

for, then the integral in (2.16) converges for $\operatorname{Re} \zeta>-\varepsilon / 2$ and (2.16) remains true by uniqueness of analytic continuations. Then

$$
\left\|F_{\varepsilon}^{0}(\zeta)\right\|_{L_{2}, L_{2}} \leqq \frac{1}{|\zeta|} \int_{0}^{\infty} e^{(-\operatorname{Re} \zeta-\varepsilon / 2) t} d t
$$


which yields (2.12) for $\zeta$ away from 0 . Near $\zeta=0$ there is nothing to prove since $F_{\varepsilon}^{0}(\zeta)$ is continuous.

To prove (2.17) let $\chi_{|x| \geqq t / 2}$ be the operator multiplication by the characteristic function of $\{|x| \geqq t / 2\}$ and $\chi_{|x| \leqq t / 2}$ similarly. Then

$$
\begin{aligned}
E_{\varepsilon} P(t) E_{\varepsilon} & =E_{\varepsilon} P E_{\varepsilon} \chi_{|x| \leqq t / 2}+E_{\varepsilon} P E_{\varepsilon} \chi_{|x| \geqq t / 2} \\
& =\chi_{|x| \geqq t / 2} E_{\varepsilon} P E_{\varepsilon} \chi_{|x| \leqq t / 2}+E_{\varepsilon} P E_{\varepsilon} \chi_{|x| \geqq t / 2},
\end{aligned}
$$

where Huyghen's principle is used in the second step. Choose $r_{0}>0$ so that $\varrho(x)=|x|$ for $|x| \geqq r_{0}$ and suppose that $t \geqq 2 r_{0}$ then

$$
\begin{aligned}
\left\|\chi_{|x| \geqq t / 2} E_{\varepsilon}\right\|_{L_{2}, L_{2}} & =\left\|E_{\varepsilon} \chi_{|x| \geqq t / 2}\right\|_{L_{2}, L_{2}} \\
& =\sup _{|x| \geqq t / 2} e^{-\varepsilon|x|}=e^{-\varepsilon t / 2} .
\end{aligned}
$$

This together with (2.18) proves (2.17).

Corollary 4. If $Q \in L_{p}$ with $2 \leqq p \leqq \infty$ and $\operatorname{Re} \zeta>-\varepsilon / 2$ then $Q F_{\varepsilon}^{0}(\zeta) \in \operatorname{Hom}\left(L_{2}\right)$. In addition, for any $a>0$, there is a constant $c=c(a, \varepsilon, p)$ such that

$$
\left\|Q F_{\varepsilon}^{0}(\zeta)\right\|_{L_{2}, L_{2}} \leqq c\|Q\|_{L_{p}}\left(\frac{1}{1+|\zeta|}\right)^{1-\frac{2}{p}}
$$

for $\operatorname{Re} \zeta>a-\varepsilon / 2$.

Proof. For $p=2$, (2.19) follows from (2.13) and for $p=\infty$ it follows from (2.12). Interpolating between these extreme cases yields the general result.

With the above result in hand we return to the original goal of showing that $F_{\varepsilon}(\zeta)$ is small for $\operatorname{Im} \zeta$ large.

Proposition 5. Suppose that $e^{2 \varepsilon \varrho} V \in L_{p}\left(\mathbb{R}^{3}\right)$ for some $p>2$, then for any a $>0$ there are positive constants $c_{i}=c_{i}\left(a, \varepsilon,\left\|e^{2 \varepsilon \varrho} V\right\|_{L_{p}}\right), i=1,2$ such that $F_{\varepsilon}(\zeta)$ has no poles in the region $\left\{\zeta: \operatorname{Re} \zeta>a-\varepsilon / 2\right.$ and $\left.|\zeta|>c_{1}\right\}$ and in that region

$$
\left\|F_{\varepsilon}(\zeta)\right\|_{L_{2}, L_{2}} \leqq c_{2}\left(\frac{1}{1+|\zeta|}\right) .
$$

Proof. Fix $a>0$. Equation (2.11) and Corollary 4 yield the estimate

$$
\|K(\zeta)\|_{L_{2}, L_{2}} \leqq c(a, \varepsilon)\left\|e^{2 \varepsilon \varrho} V\right\|_{L_{p}}(1+|\zeta|)^{-1+\frac{2}{p}}
$$

so we may choose $c_{1}\left(a, \varepsilon,\left\|e^{2 \varepsilon \varrho} V\right\|_{L_{p}}\right)$ so that $\|K(\zeta)\| \leqq 1 / 2$ for $\operatorname{Re} \zeta>a-\varepsilon / 2$ and $|\zeta|$ $>c_{1}$. Then (2.10) shows that $F_{\varepsilon}(\zeta)$ is holomorphic in this region and satisfies $\left\|F_{\varepsilon}(\zeta)\right\|_{L_{2}, L_{2}} \leqq 2\left\|F_{\varepsilon}^{0}(\zeta)\right\|_{L_{2}, L_{2}}$. Estimate (2.12) completes the proof.

Since $(\tau-i H)^{-1}=-i R(-i \tau)$ occurs in the representation (2.1) it will be important to translate inequality (2.20) into an estimate for $R_{\varepsilon}(-i \tau)^{1}$. We present a result needed in the proof of Theorem 1 . For $0<\beta<\varepsilon / 2$, consider the region $\mathscr{V}_{\beta}$ in $\zeta$ plane shaded in Figure 1.

\footnotetext{
1 The reader is reminded that with the convention $H_{0}=\Delta$ the resolvent $R(z)$ is singular for $z \in \mathbb{R}_{-}$.
} Thus $R(\sqrt{z})$ is singular on the imaginary axis $i \mathbb{R}$ 


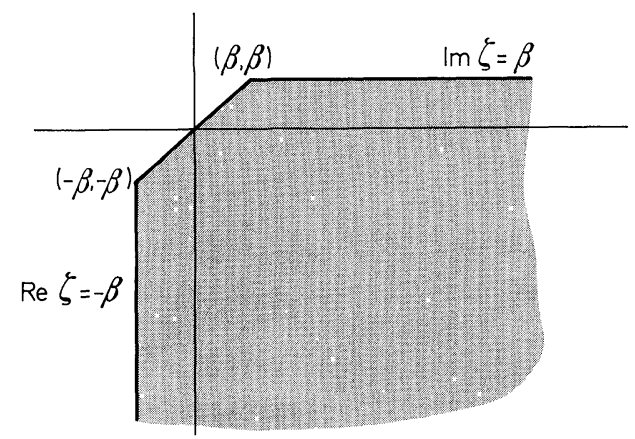

Fig. 1. The region $\mathscr{V}_{\beta}$ in the $\zeta$ plane

Under the mapping $\tau=i \zeta^{2}$ this is taken $1-1$ onto the region $\mathscr{U}_{\beta}$ shaded in Figure 2.

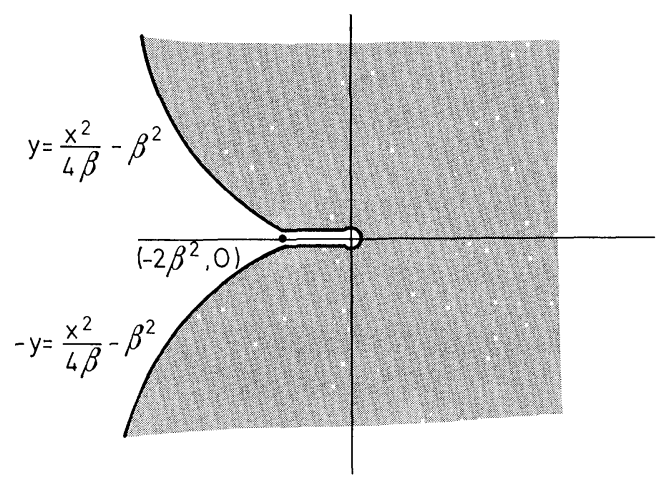

Fig. 2. The region $\mathscr{U}_{\beta}$ in the $\tau$-plane $(\tau=x+i y)$

If $\zeta \in \mathscr{V}_{\beta}$ with $\operatorname{Re} \zeta$ large (say $\operatorname{Re} \zeta>c$ ) then $\zeta^{2}$ is in the resolvent set of $H$ and for $\operatorname{such} \zeta$

$$
R_{\varepsilon}(-i \tau)=F_{\varepsilon}(\zeta)
$$

where the mapping $-i \tau=\zeta^{2}$ is implicit in this formula and those to follow. By Proposition $2, F_{\varepsilon}(\zeta)$ continues to a meromorphic function on $\mathscr{V}_{\beta}$ and it follows that $R_{\varepsilon}(-i \tau)$ has a meromorphic extension to $\mathscr{U}_{\beta}$.

Definition. Let $G_{\varepsilon}(\tau)$ be the meromorphic continuation of $-i R_{\varepsilon}(-i \tau)$ to $\mathscr{U}_{\varepsilon / 2}$.

The reason for introducing a new notation is to avoid an ambiguity in the symbol $-i R_{\varepsilon}(-i \tau)$ when $\tau$ is in $\mathscr{U}_{\beta}$ and $-i \tau$ is not in the spectrum of $H$.

Corollary 6. Suppose $e^{2 \varepsilon \varrho} V \in L_{p}$ for some $p>2$ and that $\beta>-\varepsilon / 2$. There is a constant $c=c\left(\varepsilon, \beta,\left\|e^{2 \varepsilon \varrho} V\right\|_{L_{p}}\right)$ so that

$$
\left\|G_{\varepsilon}(\tau)\right\|_{L_{2}, L_{2}} \leqq c\left(\frac{1}{|\tau|}\right) \frac{1}{2}
$$

for all $\tau \in \mathscr{U}_{\beta}$ with $|\tau|>c$. 
Proof. $G_{\varepsilon}(\tau)=-i F_{\varepsilon}(\zeta)$ where $\tau$ and $\zeta$ are related by the mapping $-i \tau=\zeta^{2}$. Since $|\tau|^{1 / 2}=|\zeta|$, the Corollary is an immediate consequence of Proposition 5.

\section{Appendix}

In this appendix we show that $\left\|F_{\varepsilon}^{0}(i x)\right\|_{L_{2}, L_{2}} \geqq$ const $|x|^{-1}$, so inequality (2.17) gives the correct rate of decay as $|\operatorname{Im} \zeta| \rightarrow \infty$. The lower bound is not needed in any of the proofs. A similarity by the operator $e^{-|x|} e^{\varrho}$ and a change of scale shows that it suffices to consider the operator $L(\zeta)$ with kernel $\left.e^{-|y|}(4 \pi)|y-x|\right)^{-1} e^{-\zeta|y-x|} e^{-|x|}$. The idea is to reduce to a one dimensional problem by considering the restriction of $L(\zeta)$ to spherically symmetric functions.

Let $\mathscr{R}: L_{2}^{\text {radial }}\left(\mathbb{R}^{3}\right) \rightarrow L_{2}^{\text {odd }}(\mathbb{R})$ be defined by $(\mathscr{R} g)(s)=\left.g\right|_{|x|=s}$ for $s \geqq 0$ and $\mathscr{R} g(-s)$ $=-\mathscr{R} g(s)$, and let $\mathscr{F}_{k}$ be the Fourier transform acting on $L_{2}\left(\mathbb{R}^{k}\right)\left(\right.$ kernel of $\mathscr{F}_{k}$ is $\left.(2 \pi)^{-k / 2} e^{-i x \cdot \xi}\right)$. Then $\mathscr{R}$ commutes with $\mathscr{F}_{k}$ in the sense that $\mathscr{F}_{k}: L_{2}^{\text {radial }}\left(\mathbb{R}^{3}\right) \rightarrow L_{2}^{\text {radial }}\left(\mathbb{R}^{3}\right), \quad \mathscr{F}_{1}: L_{2}^{\text {odd }}(\mathbb{R}) \rightarrow L_{2}^{\text {odd }}(\mathbb{R}), \quad$ and $\quad \mathscr{R}^{-1} \mathscr{F}_{1} \mathscr{R}=\mathscr{F}_{3} \quad$ on $L_{2}^{\text {radial }}\left(\mathbb{R}^{3}\right)$.

To estimate $L(\zeta)$ observe that for $\operatorname{Re} \zeta>0$

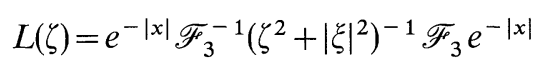

where we have used the fact that $\mathscr{F}_{3}\left((4 \pi|x|)^{-1} e^{-\zeta|x|}\right)=(2 \pi)^{-3 / 2}\left(\zeta^{2}+|\xi|^{2}\right)^{-1}$. Thus,

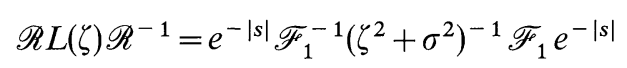

on $L_{2}^{\text {odd }}$ where we have used $s$ for the variable on $\mathbb{R}$ and $\sigma$ for the dual variable. On $L_{2}(\mathbb{R})$ the operator $\mathscr{F}_{1}^{-1}\left(\zeta^{2}+\sigma^{2}\right)^{-1} \mathscr{F}_{1}$ has kernel $\zeta^{-1} e^{-\zeta\left|s^{\prime}-s\right|}$. Thus for $\varphi, \psi \in L_{2}^{\text {odd }}$

$$
\begin{aligned}
\left(L(\zeta) \mathscr{R}^{-1} \varphi, \mathscr{R}^{-1} \psi\right)_{L_{2}\left(\mathbb{R}^{3}\right)} & =\frac{1}{\zeta} \int_{-\infty}^{\infty} \int_{-\infty}^{\infty} e^{-\left|s^{\prime}\right|} e^{-\zeta\left|s^{\prime}-s\right|} e^{-|s|} \varphi(s) \overline{\psi\left(s^{\prime}\right)} d s d s^{\prime} \\
& =\frac{1}{\zeta}\left(\iint_{s^{\prime} \geqq s}+\iint_{s^{\prime} \leqq s}\right) .
\end{aligned}
$$

Since $\varphi$ and $\psi$ are odd, the integrand is invariant under the map $\left(s, s^{\prime}\right) \mapsto-\left(s, s^{\prime}\right)$ which interchanges the regions $s^{\prime} \geqq s$ and $s^{\prime} \leqq s$. Thus the integral over $\mathbb{R}^{2}$ is twice the integral over the set $s^{\prime} \geqq s$. In this region $\left|s^{\prime}-s\right|=s^{\prime}-s$ so we obtain

$$
\left(L(\zeta) \mathscr{R}^{-1} \varphi, \mathscr{R}^{-1} \varphi\right)=\frac{2}{\zeta} \int_{s^{\prime} \geqq s} e^{-\left|s^{\prime}\right|} e^{-\zeta\left(s^{\prime}-s\right)} e^{-|s|} \varphi(s) \overline{\psi\left(s^{\prime}\right)} d s d s^{\prime} .
$$

This formula proved for $\operatorname{Re} \zeta>0$ remains true for $\operatorname{Re} \zeta>-1$ by uniqueness of analytic continuation. We restrict attention to $\zeta=i x$ for $x \in \mathbb{R}$. For such $\zeta$ the integrand in (2.24) is replaced by its complex conjugate under the mapping $\left(s, s^{\prime}\right)$ $\mapsto-\left(s, s^{\prime}\right)$. Thus for $\zeta=i x$

$$
\operatorname{Re}\left[\zeta\left(L(\zeta) \mathscr{R}^{-1} \varphi, \mathscr{R}^{-1} \psi\right)\right]=\iint_{\mathbb{R}^{2}} e^{-\left|s^{\prime}\right|} e^{-\zeta\left(s^{\prime}-s\right)} e^{-|s|} \varphi(s) \overline{\psi\left(s^{\prime}\right)} d s d s^{\prime} .
$$

Take $\varphi=\psi$ to be real valued, then Fubini's theorem and the oddness of $\varphi$ imply that

$$
\operatorname{Re}\left[\zeta\left(L(\zeta) \mathscr{R}^{-1} \varphi, \mathscr{R}^{-1} \varphi\right)\right]=4\left(\int_{0}^{\infty} e^{-s} \varphi(s) \cos \varkappa s d s\right)^{2}
$$


Let $\varphi_{\varkappa}=e^{-s} \cos \varkappa s$ for $s \geqq 0$, then the $L_{2}^{\text {odd }}(\mathbb{R})$ norm of $\varphi$ is bounded independent of $x$ and the last identity yields

$$
\operatorname{Im}\left(L(i \varkappa) \mathscr{R}^{-1} \varphi_{\varkappa}, \mathscr{R}^{-1} \varphi_{\chi}\right)=-\frac{4}{\varkappa} \int_{0}^{\infty} e^{-2 s}(\cos \varkappa s)^{2} d s .
$$

As $|x| \rightarrow \infty$, the integral on the right converges to $\frac{1}{2} \int_{0}^{\infty} e^{-2 s} d s$, so $(2.25)$ yields the desired lower bound.

\section{§3. Proof of the Main Theorem}

For $\operatorname{Re} \tau>0$ we have the identity

$$
-i R(-i \tau)=\int_{0}^{\infty} e^{-\tau t} e^{i t H} d t .
$$

Thus, $E_{\varepsilon} e^{i t H} E_{\varepsilon}$ has Laplace transform $-i R_{\varepsilon}(-i \tau)$. Our strategy is to recover $E_{\varepsilon} e^{i t H} E_{\varepsilon}$ by inverting the Laplace transform. For technical reasons, we consider $E_{\varepsilon}\left(e^{i t H}-I\right) E_{\varepsilon}$ which has Laplace transform $-i R_{\varepsilon}(-i \tau)-\tau^{-1} E_{\varepsilon}^{2}$. Formally, for any $d>0$

$$
E\left(e^{i t H}-I\right) E=\frac{1}{2 \pi i} \int_{d-i_{\infty}}^{d+i_{\infty}} e^{\tau t}\left(-i R_{\varepsilon}(-i \tau)-\frac{E^{2}}{\tau}\right) d \tau
$$

where the $\varepsilon$ dependence of $E_{\varepsilon}$ has been suppressed. The first goal is to study the convergence of the integral in (3.2).

Multiplying the identity $R(-i \tau)(-i \tau-H)=I$ on the left and the right by $E$ yields

$$
-i R_{\varepsilon}(-i \tau)-\frac{E^{2}}{\tau}=\frac{1}{\tau} E R_{\varepsilon}(-i \tau) H E .
$$

For $\psi$ in the Sobolev space $W_{2}$ the commutator of $H$ and $E$ is given by

$$
[H, E] \psi=\left[H_{0}, E\right] \psi=2\left(\operatorname{grad} e^{-\varepsilon \varrho}\right) \cdot(\operatorname{grad} \psi)+\psi\left(H_{0} e^{-\varepsilon \varrho}\right) .
$$

Since every derivative of $\varrho$ is uniformly bounded on $\mathbb{R}^{3}$ it follows that for any multiindex $\alpha,\left|D_{x}^{\alpha} e^{-\varepsilon \varrho}\right| \leqq c_{\alpha} e^{-\varepsilon \varrho}$. Therefore, $E^{-1}[H, E]$ is a bounded operator from $W_{1}\left(\mathbb{R}^{3}\right)$ to $L_{2}\left(\mathbb{R}^{3}\right)$. Let $M=H+E^{-1}[H, E] \in \operatorname{Hom}\left(W_{2}, L_{2}\right)$ then

$$
-i R_{\varepsilon}(-i \tau)-\frac{E^{2}}{\tau}=\frac{1}{\tau} R_{\varepsilon}(-i \tau) M
$$

an identity in $\operatorname{Hom}\left(W_{2}, L_{2}\right)$. It follows that

$$
\left\|-i R_{\varepsilon}(-i \tau)-\frac{E^{2}}{\tau}\right\|_{W_{2}, L_{2}} \leqq \frac{c}{|\tau|}\left\|R_{\varepsilon}(-i \tau)\right\|_{L_{2}, L_{2}}
$$

Corollary 6 shows that for $|\tau|$ large $\left\|R_{\varepsilon}(-i \tau)\right\|_{L_{2}, L_{2}} \leqq c|\tau|^{-1 / 2}$. This together with (3.5) proves the absolute convergence of the integral in (3.2), in the space $\operatorname{Hom}\left(W_{2}, L_{2}\right)$, and therefore justifies (3.2). 
Next we deform the contour in (3.2) into the left halfplane. For any $\beta>-\varepsilon / 2$ the integrand has the meromorphic continuation $e^{\tau t}\left(G_{\varepsilon}(\tau)-\tau^{-1} E^{2}\right)$ to the region $\mathscr{U}_{\beta}$. In the following computations we suppress the $\varepsilon$ dependence of $G_{\varepsilon}$. By Proposition $5, F_{\varepsilon}(\zeta)$ has only a finite number of poles in the region $\operatorname{Re} \zeta \geqq \beta$ so $G(\tau)$ has only a finite number of poles in $\overline{\mathscr{U}_{\beta}}$. We may decrease $\beta$ if necessary to insure that $F_{\varepsilon}(\zeta)$ has no poles on $\{|\zeta| \leqq \beta\} \bigcup \partial \mathscr{V}_{\beta}$ with the possible exception of 0 . Then $G(\tau)$ has no poles on $\partial \mathscr{U}_{\beta} \backslash 0$ and is continuous from above and below at all points of the "slit" $\left\{(s, 0),-\beta^{2} \leqq s<0\right\}$ in $\mathscr{U}_{\beta}$.

Identity (3.4) continues to the region $\mathscr{U}_{\varepsilon / 2}$ in the form $G(\tau)-\tau^{-1} E^{2}=\tau^{-1} G(\tau) M$ and this implies the analogue of (3.5) for $\tau \in \mathscr{U}_{\varepsilon / 2}$

$$
\left\|G(\tau)-\frac{E^{2}}{\tau}\right\|_{W_{2}, L_{2}} \leqq \frac{c}{|\tau|}\|G(\tau)\|_{L_{2}, L_{2}} .
$$

Then Corollary 6 implies that there is a $c_{1}$ so that for $\tau \in \mathscr{U}_{\beta}$ with $|\tau|>c_{1}$,

$$
\left\|G(\tau)-\frac{E^{2}}{\tau}\right\|_{W_{2}, L_{2}} \leqq c|\tau|^{-3 / 2} .
$$

Let $\mathscr{B}$ be a disc in $\mathbb{C}$ with center at the origin and radius less than $\beta^{2}$. Estimate (3.6) justifies the use of Cauchy's theorem to deform the contour $(d-i \infty, d+i \infty)$ to $\partial\left(\mathscr{U}_{\beta} \backslash \mathscr{B}\right)$.

$$
E e^{i t H} E-E^{2}=\frac{1}{2 \pi i} \int_{\partial\left(\mathcal{U}_{\beta} \backslash \mathscr{B}\right)} e^{\tau t}\left(G(\tau)-\frac{E^{2}}{\tau} d \tau+\sum_{j} \operatorname{Res}\left(e^{\tau t} G(\tau) ; \tau=\tau_{j}\right)\right.
$$

where the finite sum is over all poles of $G(\tau)$ in the region $\mathscr{U}_{\beta} \backslash 0$. The segment from $-\beta^{2}$ to radius $(\mathscr{B})$ on the $x$-axis requires some comment. It is understood that this segment is traversed twice. First from left to right with $G$ given the values $G(\tau-i 0)$, then the contour goes around the disc $\mathscr{B}$ and returns on the $x$ axis with $G$ given the values $G(\tau+i 0)$. The contour $\partial\left(\mathscr{U}_{\beta} \backslash \mathscr{B}\right)$ consists of a bounded arc in $\operatorname{Re} \tau \geqq \beta^{2}$ and the part in $\operatorname{Re} \tau<\beta^{2}$. On the first piece the terms of the integrand are continuous with values in $\operatorname{Hom}\left(L_{2}\right)$ so that part of the integral converges absolutely in $\operatorname{Hom}\left(L_{2}\right)$. On the unbounded part there is a constant $c>0$ so that

$$
\operatorname{Re} \tau \leqq-c\left(1+|\operatorname{Im} \tau|^{1 / 2}\right) .
$$

Since Corollary 6 implies that $\|G\|_{L_{2}, L_{2}}$ is bounded on $\partial\left(\mathscr{U}_{\beta} \backslash \mathscr{B}\right)$ it follows that both

$$
\int_{\partial\left(\mathscr{U}_{\beta} \backslash \mathscr{B}\right)} e^{\tau t} G(\tau) d \tau \text { and } \quad \frac{-1}{2 \pi i} \int_{\partial\left(\mathscr{U}_{\beta} \backslash \mathscr{B}\right)} e^{\tau t} \frac{E^{2}}{\tau} d \tau
$$

are absolutely convergent in $\operatorname{Hom}\left(L_{2}\right)$. In addition, the second expression can be evaluated exactly. It is $-E^{2}$. Thus

$$
E e^{i t H} E=\frac{1}{2 \pi i} \int_{\partial\left(\mathscr{U}_{\beta} \backslash \mathscr{B}\right)} e^{\tau t} G(\tau) d \tau+\sum_{j} \operatorname{Res}\left(e^{\tau t} G(\tau) ; \tau=\tau_{j}\right)
$$

the integral converging absolutely in $\operatorname{Hom}\left(L_{2}\right)$.

The theorem is proved by studying the terms in (3.8). Partition the poles into two classes, those on the imaginary axis, $i \omega_{1}, i \omega_{2}, \ldots, i \omega_{l}$ and those with $\operatorname{Re} \tau_{j}<0$. 
The residue at a pole with negative real part is of the form

$$
\operatorname{Res}\left(G(\tau) e^{\tau t} ; \tau=\tau_{j}\right)=p_{j}(t) e^{\tau} j^{t}
$$

where $p_{j}$ is a polynomial in $t$ with compact operator coefficients and degree $\left(p_{j}\right)$ is one less than the order of the pole. The operators (3.9) decay exponentially as $t \rightarrow \infty$. For the imaginary poles abserve that the dissipativity of $i H$ implies that $\|R(i \tau)\|_{L_{2}, L_{2}} \leqq(\operatorname{Re} \tau)^{-1}$ so

$$
\|G(\tau)\|_{L_{2}, L_{2}} \leqq \frac{1}{\operatorname{Re} \tau} \text { for } \operatorname{Re} \tau>0 .
$$

It follows that the poles $i \omega_{j}$ must be simple,

$$
G(\tau)=\frac{A_{j}}{\tau-i \omega_{j}}+\text { function analytic at } i \omega_{j}
$$

where $A_{j}$ is compact. It follows that

$$
\operatorname{Res}\left(e^{\tau t} G(\tau) ; \tau=i \omega_{j}\right)=e^{i \omega_{j} t} A_{j} .
$$

Consider next, the integral in (3.8). The contour is composed of two parts, the parabolic arcs in the region $\operatorname{Re} \tau \leqq-\beta^{2}$ and the thermometer shaped contour in $\operatorname{Re} \tau \geqq-\beta^{2}$. On the parabolic arcs $\|G(\tau)\|_{L_{2}, L_{2}}$ is bounded. Then, the estimate (3.7) for $\operatorname{Re} \tau$ implies that for $t \geqq 1$ the norm in $\operatorname{Hom}\left(L_{2}\right)$ of the integral over the parabolic arcs is $O\left(e^{-c t}\right)$. This leaves the integral over the thermometer. The number $\beta$ was chosen so that $F_{\varepsilon}$ has no poles $\zeta$ of modulus $0<|\zeta| \leqq \beta$ so the Laurent expansion

$$
F_{\varepsilon}(\zeta)=\sum_{j=-N}^{\infty} B_{j} \zeta^{j}
$$

converges on a punctured disc of radius larger than $\beta$. Inequality (3.10) shows that for $\zeta \in \mathbb{R}_{+},\left\|F_{\varepsilon}(\zeta)\right\|_{L_{2}, L_{2}} \leqq \zeta^{-2}$ so that $N \leqq 2$. Let $\tau \mapsto \sqrt{i \tau}$ be the inverse of the map $\zeta \mapsto \zeta^{2}$ from $\mathscr{V}_{\varepsilon / 2}$ to $\mathscr{U}_{\varepsilon / 2}$ then for $|\tau| \leqq \beta^{2}, \tau \in \mathscr{U}_{\beta}$,

$$
\begin{aligned}
& G(\tau)=-i \sum_{j=-2}^{\infty} B_{j}(\sqrt{-i \tau})^{j}=\frac{B_{-2}}{\tau}+C(\tau) \\
& \|C(\tau)\|_{L_{2}, L_{2}} \leqq c|\tau|^{-1 / 2} .
\end{aligned}
$$

Use (3.12) to evaluate the integral over the thermometer, shaped contour, $\mathscr{T}$,

$$
\frac{1}{2 \pi i} \int_{\mathscr{T}} G(\tau) e^{\tau t} d \tau=\frac{1}{2 \pi i} \int_{\mathscr{T}} \frac{B_{-2}}{\tau} e^{\tau t} d \tau+\frac{1}{2 \pi i} \int_{\mathscr{T}} C(\tau) e^{\tau t} d \tau
$$

Then first integral on the right is exactly equal to $B_{-2}$. By (3.13) and Cauchy's theorem, the circular arc in the $C(\tau)$ term can be shrunk to the origin. Then by (3.13) again

$$
\begin{aligned}
\left\|\int_{\mathscr{T}} C(\tau) e^{\tau-t} d \tau\right\| & \leqq 2 c \int_{0}^{\beta^{2}} \sigma^{-1 / 2} e^{-\sigma t} d \sigma \\
& \leqq 2 c \int_{0}^{\infty} \sigma^{-1 / 2} e^{-\sigma t} d \sigma=2 c t^{-1 / 2} \Gamma(1 / 2) .
\end{aligned}
$$


Summarizing the analysis of (3.8) we have shown that the residues at poles with negative real part and the integral over the parabolic arcs are both $O\left(e^{-c t}\right)$ for $c>0$. The residues at imaginary poles are given by (3.11) and the thermometer integral is $B_{-2}+O\left(t^{-1 / 2}\right)$. Thus we have shown that

$$
\left\|E_{\varepsilon} e^{i t H} E_{\varepsilon}-\left(B_{-2}+\sum_{j=1}^{l} e^{i \omega_{j} t} A_{j}\right)\right\|_{L_{2}, L_{2}} \leqq c(1+t)^{-1 / 2} .
$$

To symmetrize this formula let $\omega_{0}=0$ and $A_{0}=B_{-2}$. A consequence of (3.14) is that for $j=0,1, \ldots, l$

$$
\text { strong }-\lim \frac{1}{T} \int_{0}^{T} e^{-i \omega_{j} t} E_{\varepsilon} e^{i H t} E_{\varepsilon} d t=A_{j},
$$

and

$$
\text { strong }-\lim \frac{1}{T} \int_{0}^{T} e^{-i \omega t} E_{\varepsilon} e^{i t H} E_{\varepsilon} d t=0
$$

if $\omega \neq \omega_{j}$ for some $j$. The mean ergodic theorem asserts that

$$
\text { strong }-\lim \frac{1}{T} \int_{0}^{T} e^{-i \omega t} e^{i H t} d t=\Pi_{\omega}
$$

where $\Pi_{\omega}$ is orthogonal projection onto nullspace $(H-i \omega)$. Thus, in the notation of Theorem 1

$$
\Pi_{B}=\sum_{j=0}^{l} \Pi_{i \omega_{j}} \text { and } E_{\varepsilon} \Pi_{i \omega_{j}} E_{\varepsilon}=A_{j} .
$$

Now

$$
e^{i t H} \Pi_{S}=e^{i t H}\left(I-\Pi_{B}\right)=e^{i t H}-\sum_{j=0}^{\infty} e^{i \omega_{j} t} \Pi_{i \omega_{j}} .
$$

Thus using (3.17) we see that (3.14) is exactly the estimate (1.8) of Theorem 1.

The invariance of $\mathscr{H}_{B}$ under $e^{i t H}$ is immediate, and, for any $t \geqq 0$ the contractions $e^{i t H}$ are unitary on $\mathscr{H}_{B}$. The invariance of $\mathscr{H}_{S} \equiv\left(\mathscr{H}_{B}\right)^{\perp}$ is a simple consequence of these facts ([11, Proposition 6]). Alternatively, the estimate (1.8) implies that

$$
\mathscr{H}_{S}=\left\{\psi \in L_{2}: \text { as } t \rightarrow \infty, e^{i t H} \psi \rightarrow 0 \text { in } L_{2}^{\text {loc }}\left(\mathbb{R}^{3}\right)\right\}
$$

and the invariance is apparent. This completes the proof of Theorem 1.

\section{§4. Genericity of $t^{-3 / 2}$ Decay}

In this section (3.8) is analysed more closely. We obtain an asymptotic expansion for $E_{\varepsilon} e^{i t H} \Pi_{S} E_{\varepsilon}$ in decreasing negative powers of $t$. Necessary and sufficient conditions are given for the scattering solutions to decay locally at a rate $t^{-3 / 2}$. It turns our that $t^{-3 / 2}$ decay is generic. On the other hand, $t^{-1 / 2}$ decay 
occurs at those values of the coupling constant when an eigenvalue or resonance emerges from the origin, in particular, if nullspace $(H) \neq\{0\}$.

Consider the integral over the thermometer shaped part of the contour $\partial\left(\mathscr{U}_{\beta} \backslash \mathscr{B}\right)$,

$$
\frac{1}{2 \pi i} \int_{\mathscr{T}} G(\tau) e^{\tau t} d \tau
$$

Since the expansion (3.12) converges uniformly on $|\tau| \leqq \beta^{2}$ we may integrate term by term to obtain

$$
B_{-2}+\sum_{j \geqq-1} \frac{-i B_{2}}{2 \pi i} \int_{\mathscr{T}}(-i \tau)^{j / 2} e^{\tau t} d \tau
$$

Since $j \geqq-1$ the circular part of the contour may be shrunk to the origin. In addition, if $j$ is even then the integrand is analytic in the disc $|\tau| \leqq \beta^{2}$ so the integral vanishes. For $j$ odd and $\tau \in\left[-\beta^{2}, 0\right],(-i(\tau+i 0))^{j / 2}=-(i(\tau-i 0))^{j / 2}$ and the integral in (4.2) is equal to

$$
-2 e^{i \pi / 4} \int_{0}^{\beta^{2}} \sigma^{j / 2} e^{-\sigma t} d t=-2 t^{-\frac{j}{2}-1} e^{i \pi / 4} \int_{0}^{\beta^{2} t} \sigma^{j / 2} e^{-\sigma} d \sigma .
$$

Let

$$
a_{j}=\frac{1}{\pi} e^{i \pi / 4} \int_{0}^{\infty} \sigma^{j / 2} e^{-\sigma} d \sigma=\frac{e^{i \pi / 4}}{\pi} \Gamma\left(\frac{j}{2}+1\right)
$$

then it follows that the integral (4.1) has the asymptotic expansion

$$
B_{-2}+\sum_{k=0}^{\infty} a_{2 k-1} B_{2 k-1} t^{-k-1 / 2}
$$

in the sense that for any $N$ there is a $c>0$ so that

$$
\left\|\frac{1}{2 \pi i} \int_{\mathscr{T}} G(\tau) e^{\tau t} d \tau-\left(B_{-2}+\sum_{0}^{N} a_{2 k-1} B_{2 k-1} t^{-k-\frac{1}{2}}\right)\right\|_{L_{2}, L_{2}} \leqq c t^{-N-3 / 2} .
$$

Since the contribution of the rest of $\partial\left(\mathscr{U}_{\beta} \backslash \mathscr{B}\right)$ to the integral in (3.8) is $O\left(e^{-c t}\right)$ this proves the following result.

Theorem 2. Let $H$ and $V$ be as in Theorem $1, F_{\varepsilon}(\zeta)$ be the meromorphic continuation of $R_{\varepsilon}\left(\zeta^{2}\right)$ to $\operatorname{Re} \zeta>-\varepsilon, B_{j}, j \geqq-2$ be the coefficients of the Laurent expansion of $F_{\varepsilon}$ at $\zeta=0$, and $a_{j}$ be given by (4.3). Then for any integer $N>0$ there is a constant $c$ so that for all $t>0$

$$
\left\|E_{\varepsilon} e^{i t H} \Pi_{S} E_{\varepsilon}-\sum_{k=0}^{N} a_{2 k-1} B_{2 k-1} t^{-k-\frac{1}{2}}\right\|_{L_{2}, L_{2}} \leqq c t^{-N-\frac{3}{2}} .
$$

A particularly important role is played by the case $N=0$,

$$
\left\|E_{\varepsilon} \varepsilon^{i t H} \Pi_{S} E_{\varepsilon}-a_{-1} t^{-1 / 2} B_{-1}\right\|_{L_{2}, L_{2}} \leqq c t^{-3 / 2} .
$$


This shows that it is $B_{-1}$ which determines whether the scattering solutions decay with rate $t^{-1 / 2}$ or $t^{-3 / 2}$. There is $t^{-3 / 2}$ decay if and only if $B_{-1}=0$. For "most" potentials $F_{\varepsilon}(\zeta)$ is analytic in a neighborhood of $\zeta=0$ so, in particular, $B_{-1}=0$. In this way we show that generically there is $t^{-3 / 2}$ decay. The following proposition is needed in the proof and gives a simple criterion for the regularity of $F_{\varepsilon}(\zeta)$ at the origin.

Proposition 7. Suppose that $e^{\varepsilon \varrho} V \in L_{2}$, that $F_{\varepsilon}(\zeta)$ is the meromorphic continuation of $R_{\varepsilon}\left(\zeta^{2}\right)$ to $\operatorname{Re} \zeta>-\varepsilon$, and that $K(\zeta)$ is the compact operator with kernel (2.8). Then $F_{\varepsilon}(\zeta)$ has a pole at $\zeta=0$ if and only if $I-K(0)$ is not invertible.

Proof. Identity (2.9) when analytically continued reads

$$
F_{\varepsilon}(\zeta)=F_{\varepsilon}^{0}(\zeta)[I+K(\zeta)]^{-1} .
$$

If $[I+K(0)]^{-1}$ exists then $[I+K(\zeta)]^{-1}$ is analytic on a neighborhood of $\zeta=0$. Since $F_{\varepsilon}^{0}(\zeta)$ is analytic on $\operatorname{Re} \zeta>-\varepsilon$ it follows that $F_{\varepsilon}(\zeta)$ is regular at $\zeta=0$.

Conversely, if $I+K(0)$ is not invertible then $[I+K(\zeta)]^{-1}$ has a pole at $\zeta=0$. Consider the Laurent expansion

$$
[I+K(\zeta)]^{-1}=K_{-N} \zeta^{-N}+K_{-N+1} \zeta^{-N+1}+\ldots
$$

with $K_{-N} \neq 0$. Then

$$
F_{\varepsilon}(\zeta)=F_{\varepsilon}^{0}(0) K_{-N} \zeta^{-N}+0\left(\zeta^{-N+1}\right)
$$

so to show that $F_{\varepsilon}$ has a pole at $\zeta=0$ it suffices to show that $F_{\varepsilon}^{0}(0) K_{-N} \neq 0$. Thus it suffices to show that nullspace $\left(F_{\varepsilon}^{0}(0)\right)=\{0\}$. Now $F_{\varepsilon}^{0}(0)$ has kernel $e^{-\varepsilon \varrho(y)}(4 \pi \mid y$ $-x \mid)^{-1} e^{-\varepsilon \varrho(x)}$ so far $\psi \in L_{2}$

$$
\left(F_{\varepsilon}^{0}(0) \psi, \psi\right)_{L_{2}}=\frac{1}{4 \pi} \iint \frac{\varphi(x) \overline{\varphi(y)}}{|y-x|} d x d y=\int \frac{|\hat{\varphi}(\xi)|^{2}}{|\xi|^{2}} d \xi
$$

where $\varphi=e^{-\varepsilon \varrho} \psi$, and $\hat{\varphi} \in C^{\infty}\left(\mathbb{R}^{3}\right) \cap L_{2}$ is the Fourier transform of $\varphi$. It follows that if $F_{\varepsilon}^{0}(0) \psi=0$ then $\varphi=0$ so $\psi=e^{\varepsilon \varrho} \varphi=0$ so nullspace $\left(F_{\varepsilon}^{0}(0)\right)=\{0\}$ and the proof is complete.

Example. Dolph, McLeod, and Thoe [2, p. 332] construct examples of continuous real potentials $V$ with compact support for which $I+K(0)$ is not invertible but nullspace $(H)=\{0\}$. By Proposition $7, F_{\varepsilon}(\zeta)$ has a pole at $\zeta=0$ and the zero nullspace implies that $B_{-2}=0$. It follows that $B_{-1} \neq 0$ so these examples exhibit $t^{-1 / 2}$ decay. Notice that the nontrivial "if" part of Proposition 7 used here.

Theorem 3. (1) If $z \mapsto V_{z}$ is a holomorphic function on a connected open set $\Omega \subset \mathbb{C}$ with values in $L_{2}\left(\mathbb{R}^{3}, e^{2 \varepsilon \varrho(x)} d x\right)$ and $V_{z_{0}}=0$ for some $z_{0} \in \Omega$ then for all but a discrete set of $z$ the meromorphic continuation of $E_{\varepsilon}\left(\zeta^{2}-H_{0}-V_{z}\right)^{-1} E_{\varepsilon}$ does not have a pole at $\zeta=0$. In particular if $H_{g}=H_{0}+g V$ with $V$ as in Theorem 2 and $g \in \mathbb{R}$, then, for all but a discrete set of values of $g,\left\|E_{\varepsilon} e^{i t H} \Pi_{S} E_{\varepsilon}\right\|_{L_{2}, L_{2}}=O\left(t^{-3 / 2}\right)$.

(2) If $e^{-\varepsilon \varrho} V \in L_{2}$ and $F_{\varepsilon}(\zeta)$ is regular at $\zeta=0$ then there is a neighborhood of $V$ in $L_{2}\left(\mathbb{R}^{3}, e^{2 \varepsilon \varrho(x)} d x\right)$ and $a \delta>0$ such that for all $V^{\prime}$ in this neighborhood, the analytic continuation of $E_{\varepsilon}\left(\zeta^{2}-H_{0}-V\right)^{-1} E_{\varepsilon}$ is regular for $|\zeta|<\delta$. 
Proof of (1). Let $K_{z}$ have kernel

$$
\frac{e^{\varepsilon \varrho(y)} V_{z}(y) e^{-\varepsilon \varrho(x)}}{4 \pi|y-x|} .
$$

Then $z \mapsto K_{z}(y, x)$ is holomorphic on $\Omega$ with values in $L_{2}\left(\mathbb{R}^{3} \times \mathbb{R}^{3}\right)$ so the map $z$ $\mapsto K_{z}$ is holomorphic on $\Omega$ with values in the compact operators on $L_{2}$. By Proposition 7, $E_{\varepsilon}\left(\zeta^{2}-H_{0}-V_{z}\right)^{-1} E_{\varepsilon}$ is regular at $\zeta=0$ if and only if $I+K_{z}(0)$ is invertible. Since $I+K_{z_{0}}(0)=I$ is invertible, the analytic Fredholm theorem implies that the inverse exists for all but a discrete set of $z$.

Proof of (2). Let $K(\zeta)$ be as in Proposition 7 and $K^{\prime}(\zeta)$ the analogous operator with $V^{\prime}$ instead of $V$.

Then there is a constant $c_{1}>0$ so that

$$
\left\|K(0)-K^{\prime}(0)\right\|_{\text {Hilbert-Schmidt }} \leqq c_{1}\left\|V-V^{\prime}\right\|_{L_{2}\left(\mathbb{R}^{3}, e^{2 \varepsilon e} d x\right)} .
$$

In addition, for $|\zeta|<\varepsilon / 2$ there is a constant $c_{2}>0$ so that

$$
\left\|K^{\prime}(\zeta)-K^{\prime}(0)\right\|_{\text {Hilbert-Schmidt }} \leqq c_{2}|\zeta| \text {. }
$$

Thus, there is a neighborhood of $V$ in $L_{2}\left(\mathbb{R}^{3}, e^{2 \varepsilon e} d x\right)$ and a $\delta>0$ so that for $V^{\prime}$ in the neighborhood and $|\zeta|<\delta$,

$$
\left\|K^{\prime}(\zeta)-K(0)\right\|_{L_{2}, L_{2}}\left\|[I+K(0)]^{-1}\right\|_{L_{2}, L_{2}}<1 .
$$

The Neumann series expansion implies that for such $V^{\prime},\left[I+K^{\prime}(\zeta)\right]^{-1}$ is holomorphic for $|\zeta|<\delta$. By $(4.6), F_{\varepsilon}^{\prime}(\zeta)$ is regular for these $\zeta$.

Example. Suppose that $\operatorname{Im} V \geqq 0$ and $H_{g}=H_{0}+g V$ has a real eigenvalue $\omega(g)$ with $\omega(g) \rightarrow 0$ as $g$ decreases to $g_{0}$. Then $F_{\varepsilon, g}(\zeta)=E_{\varepsilon}\left(\zeta^{2}-H_{0}-g V\right)^{-1} E_{\varepsilon}$ has a pole at $i \omega(g)$. It follows from Part 2 of Theorem 3 that $E_{\varepsilon}\left(\zeta^{2}-H_{0}-g_{0} V\right)^{-1} E_{\varepsilon}$ has a pole at $\zeta=0$, for, if it were regular then $F_{\varepsilon, g}(\zeta)$ would be regular for $|\zeta|<\delta$ and $\left|g-g_{0}\right|$ small. If $V$ is not very oscillatory then there will be no negative eigenvalues of $H_{g}$ so that $\omega(g)$ emerges from the continuous spectrum $(-\infty, 0]$ at the threshold value $g_{0}$ of the coupling constant, and we have show that $F_{\varepsilon, g}$ has a pole at $\zeta=0$ for such values of $g$. The same argument shows that there is a pole at $\zeta=0$ if there are, possibly complex, sequences $g_{n} \rightarrow g_{0}$ and $\tau_{n} \rightarrow 0$ such that $\tau_{n}$ is a pole of $F_{\varepsilon, g_{n}}$. In Section 5 we interpret such poles as resonances so the above remarks are summarized by the statement:

$F_{\varepsilon, g_{0}}$ has a pole at $\zeta=0$ for those values of $g_{0}$ at which an eigenvalue or resonance emerges from the origin.

The above example only demonstrates that there is a pole at $\zeta=0$. It is conceivable that the Laurent expansion at the pole has $B_{-2} \neq 0$ and $B_{-1}=0$ so that there would be $t^{-3 / 2}$ decay. By a more careful analysis of the dissipative case we can show that this is not possible so that when $F_{\varepsilon}$ has a pole at $\zeta=0$, the local decay is at a rate $t^{-1 / 2}$. Thus at threshold values of the coupling constant scattering solutions decay at this show rate. The analysis also shows that $B_{-1}$ is a finite rank operator so that by imposing a finite number of conditions on the initial data $u(0), t^{-3 / 2}$ decay can be recovered. 
Theorem 4. Suppose that $V$ is as in Theorem $1, F_{\varepsilon}(\zeta)$ is the meromorphic continuation of $E_{\varepsilon}\left(\zeta^{2}-H_{0}-V\right)^{-1} E_{\varepsilon}$, and, $B_{j}$ for $j \geqq-2$ are the coefficients of the Laurent expansion of $F_{\varepsilon}$ at $\zeta=0$. If $F_{\varepsilon}$ has a pole at $\zeta=0$ then $B_{-1}$ is a nonzero operator of finite rank.

Proof. Let $K(\zeta)$ be the operator with kernel (2.9), then (4.6) implies $F_{\varepsilon}(\zeta)[I+K(\zeta)]$ $=F_{\varepsilon}^{0}(0)$. Expanding in a Laurent series about $\zeta=0$ and equating the $\zeta^{-1}$ terms yields

$$
B_{-1}[I+K(0)]+B_{-2} \frac{\partial K}{\partial \zeta}(0)=0 .
$$

It follows that range $B_{-1}[I+K(0)]$ Crange $B_{-2}$. If $\Pi$ is orthogonal projection on nullspace $\left(H_{0}+V\right)$ then (3.17) implies that $B_{-2}=E_{\varepsilon} \Pi E_{\varepsilon}$ so range $B_{-2}$ is finite dimensional. Since range $[I+K(0)]$ has finite codimension it follows that range $B_{-1}$ is finite dimensional.

It remains to show that if $B_{-1}=0$ then $F_{\varepsilon}$ is regular at $\zeta=0$. By (4.7) if $B_{-1}=0$ then $B_{-2} \frac{\partial K}{\partial \zeta}(0)=0$. From the explicit expressions for $B_{-2}$ and $K(\zeta)$ it follows that $B_{-2} \frac{\partial K}{\partial \zeta}(0)=(4 \pi)^{-1} E_{\varepsilon} \Pi V E_{\varepsilon}$. Since $E_{\varepsilon}$ has dense range and trivial nullspace, this operator vanishes if and only if $\Pi V=0$. Now, as operators defined on $\mathscr{D}\left(H_{0}\right)$,

$$
\Pi H_{0}=\Pi\left(H_{0}+V\right)-\Pi V=-\Pi V .
$$

Thus, $\Pi V$ vanishes if and only if $\Pi H_{0}=0$. Since $H_{0}$ has dense range this is equivalent to $\Pi=0$. Summarizing the above computations we have shown that

$$
B_{-2} \frac{\partial K}{\partial \zeta}(0)=0 \Leftrightarrow \Pi=0 \Leftrightarrow B_{-2}=0 .
$$

Thus, if $B_{-1}=0$ it follows that $B_{-2}=0$, and consequently that $F_{\varepsilon}$ is regular at $\zeta=0$. The proof of Theorem 4 is complete.

The next result, suggested by $\mathrm{H}$. Brezis, shows that if $V \leqq 0$ then $F(\zeta)$ is regular at $\zeta=0$ and we have $t^{-3 / 2}$ decay.

Theorem 5. Suppose, in addition to the hypothesis of Theorem 1, that $V$ is real valued and $V \leqq 0$. Then $B_{-1}=0$.

Proof. It suffices (using Proposition 7) to show that $I-K(0)$ is invertible. By Fredholm's theorem it suffices to show that $u \in L_{2}\left(\mathbb{R}^{3}\right)$ and $u=K(0) u$ imply $u=0$. If $u \in L_{2}\left(\mathbb{R}^{3}\right)$ and $u=K(0) u$ then $w=\frac{1}{4 \pi r} *\left(e^{-\varepsilon \varrho} u\right)$ satisfies $(\Delta+V) w=0$. In addition, it is not difficult to show that $w$ is a continuous function on $\mathbb{R}^{3}$ with $\lim _{|x| \rightarrow \infty} w(x)=0$.

Using the maximum principle for the Laplace operator we show that $w=0$. Suppose, on the contrary, that $\max w=w\left(x_{0}\right)>0$. Near $x_{0}$ we have $\Delta w=-V w \geqq 0$. It follows from the maximum principle that $w(x) \equiv w\left(x_{0}\right)$ for $x$ near $x_{0}$ so the closed 
set $\left\{x: w(x)=w\left(x_{0}\right)\right\}$ is also open. Therefore, we must have $w(x) \equiv w\left(x_{0}\right)$ for all $x \in \mathbb{R}^{3}$. Since $w(x) \rightarrow 0$ as $|x| \rightarrow \infty$ this provides a contradiction. In the same manner one shows that $w \leqq 0$ so, in total, $w \equiv 0$. Then $0=\Delta w=-e^{-\varepsilon \varrho} u$ so $u=0$ and the proof is complete.

\section{§5. Resonances and Poles of the Resolvent}

Consider again the identity (3.8) which holds for all $t>0$. The proof of Theorem 1 shows that integral over the parabolic arcs on $\partial \mathscr{U}_{\beta}$ is $O\left(e^{-c t}\right)$ for any $c<\beta^{2}$. Thus

$$
E e^{i t H} E=\sum_{0}^{l} e^{i \omega_{j} t} E \Pi_{i \omega_{j}} E+\sum p_{j}(t) e^{\tau_{j} t}+\frac{1}{2 \pi i} \int_{\mathscr{T}} e^{\tau t} G(\tau) d \tau+O\left(e^{c t}\right) .
$$

Recall that $\beta$ was chosen to be any number so that the modulus of each nonzero pole of $G_{\varepsilon}$ is at least $\beta^{2}$.

For Theorem 1, we considered the limit $t \rightarrow \infty$ where the exponentially decaying terms are neglibigle. However, for moderate sized time there is no reason to discard any of the terms.

The point is not that the other terms are small but simply that for times neither very large nor very small, one will observe motions with time dependence $t^{\text {power }} e^{\operatorname{Re} \tau_{j} t}$. The connection between such behavior and the poles of the analytic continuation of the resolvent (or of matrix elements of the resolvent) is an established part of the lore of theoretical physics (see [3, Chapter 8]). They occur in different contexts as unstable or virtual particles and are often called resonances. The emphasis on moderate sized time agrees with the interpretation of resonances given by Simon in [15].

\section{References}

1. Agmon, S. : Spectral properties of Schrödinger operators and scattering theory. Ann. Scuola Norm. Sup. Pisa, Series IV, 2, 151-218 (1975)

2. Dolph,C., McLeod,J., Thoe,D.: The analytic continuation of the resolvent kernel and scattering operator associated with the Schrödinger operator. J. Math. Anal. Appl. 16, 311-332 (1966)

3. Goldberger,G.M., Watson,K.: Collision theory. New York: Wiley 1964

4. Herbst, I.: Unitary equivalence of Stark hamiltonians. (To appear)

5. Hunziker,W.: The Schrödinger eigenvalue problem for $N$-particle systems. Acta Phys. Austriaca (to appear)

6. Kato,T.: Perturbation theory for linear operators. Berlin-Heidelberg-New York: Springer 1966

7. Kato, T.: Wave operators and similarity for some non-selfadjoint operators. Math. Ann. 162, 258279 (1966)

8. Lavine, R.: Constructive estimates in quantum scattering. (To appear)

9. Lax,P., Phillips, R.: Scattering theory. Rocky Mtn. J. Math. 1, 173-223 (1971)

10. Lax,P., Phillips, R. : Scattering theory for dissipative hyperbolic systems. J. Funct. Anal. 14, 172235 (1973)

11. Rauch,J.: Asymptotic behavior of solutions to hyperbolic partial differential equations with zero speeds. Comm. Pure Appl. Math. (To appear)

12. Reed,M., Simon, B.: Methods of modern mathematical physics, Vol. I: Functional analysis. New York: Academic Press 1972

13. Reed,M., Simon, B.: Methods of mathematical physics, Vol. III : Scattering theory. New York: Academic Press (to be published) 
14. Simon, B.: Quantum mechanics for Hamiltonians defined as quadratic forms. Princeton, N.J.: Princeton University Press 1971

15. Simon, B.: Resonances in $n$-body quantum systems with dilation analytic potentials and the foundations of time dependent perturbation theory. Ann. Math. 97, 247-274 (1973)

16. Vainberg, B.: On the short wave asymptotic behavior of solutions of stationary problems and the asymptotic behavior as $t \rightarrow \infty$ of solutions of nonstationary problems. Russ. Math. Surv. 30, $1-58$ (1975)

Communicated by J. Ginibre

Received October 17, 1977; in revised form January 23, 1978 